\title{
In silico identification of off-target pesticidal dsRNA binding in honey bees (Apis mellifera)
}

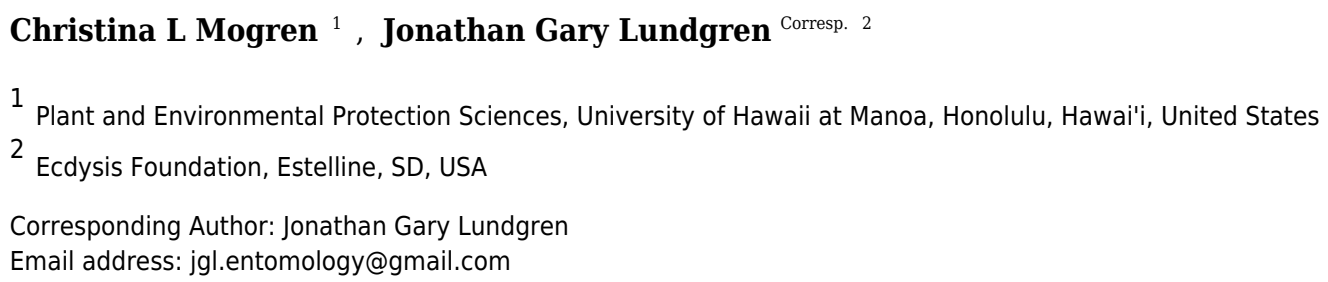

Background. Pesticidal RNAs that silence critical gene function have great potential in pest management, but the benefits of this technology must be weighed against non-target organism risks. Methods. Published studies that developed pesticidal double stranded RNAs (dsRNAs) were collated into a database. The target gene sequences for these pesticidal RNAs were determined, and the degree of similarity with sequences in the honey bee genome were evaluated statistically. Results. We identified 101 insecticidal RNAs sharing high sequence similarity with genomic regions in honey bees. The likelihood that off-target sequences were similar increased with the number of nucleotides in the dsRNA molecule. The similarities of non-target genes to the pesticidal RNA was unaffected by taxonomic relatedness of the target insect to honey bees, contrary to previous assertions. Gene groups active during honey bee development had disproportionately high sequence similarity with pesticidal RNAs relative to other areas of the genome. Discussion. Although sequence similarity does not itself guarantee a significant phenotypic effect in honey bees by the primary dsRNA, in silico screening may help to identify appropriate experimental endpoints within a risk assessment framework for pesticidal RNAi. 
1 In silico identification of off-target pesticidal dsRNA binding in honey bees (Apis mellifera)

2 Short title: Identification of off-target RNAi in honey bees

3 Christina L. Mogren ${ }^{1,2}$ and Jonathan Gary Lundgren ${ }^{1,3, *}$

$4{ }^{1}$ USDA-ARS North Central Agricultural Research Laboratory, 2923 Medary Avenue, Brookings,

5 SD, USA, 57006

$6{ }^{2}$ Current address:

7 Plant and Environmental Protection Sciences

8 University of Hawai'i at Mānoa

9 Honolulu, HI, 96822

$10{ }^{3}$ Current address:

11 Ecdysis Foundation

$1246958188^{\text {th }} \mathrm{St}$

13 Estelline, SD 57234 USA

14 *Address correspondence to:

15 Dr. Jonathan Lundgren

16 Ecdysis Foundation

$1746958188^{\text {th }}$ Street

18 Estelline, SD, USA, 57234

19 Ph: 605-695-9878

20 E-mail: jgl.entomology@gmail.com 


\section{Abstract}

22 Background. Pesticidal RNAs that silence critical gene function have great potential in pest

23 management, but the benefits of this technology must be weighed against non-target organism

24 risks.

25 Methods. Published studies that developed pesticidal double stranded RNAs (dsRNAs) were

26 collated into a database. The target gene sequences for these pesticidal RNAs were determined,

27 and the degree of similarity with sequences in the honey bee genome were evaluated statistically.

28 Results. We identified 101 insecticidal RNAs sharing high sequence similarity with genomic

29 regions in honey bees. The likelihood that off-target sequences were similar increased with the

30 number of nucleotides in the dsRNA molecule. The similarities of non-target genes to the

31 pesticidal RNA was unaffected by taxonomic relatedness of the target insect to honey bees,

32 contrary to previous assertions. Gene groups active during honey bee development had

33 disproportionately high sequence similarity with pesticidal RNAs relative to other areas of the

34 genome.

35 Discussion. Although sequence similarity does not itself guarantee a significant phenotypic effect

36 in honey bees by the primary dsRNA, in silico screening may help to identify appropriate

37 experimental endpoints within a risk assessment framework for pesticidal RNAi. 
The potential to silence critical gene function in pest species has led to the proposed

40 application of RNA interference (RNAi) as a novel class of agricultural products (Price and

41 Gatehouse 2008; Gu and Knipple 2013) that target several species of economically important

42 pests (Baum et al. 2007; Maori et al. 2009; Desai et al. 2012; Hajeri et al. 2014; Marr et al. 2014).

43 These RNAi-based pesticides may be delivered to the target pest via a number of methods,

44 including transgenic plants, diet-incorporated suspensions, and topically applications of naked or

45 encapsulated small RNAs, which elicit post-transcriptional gene silencing following ingestion.

46 Once ingested, the insect's cellular machinery cleaves long double stranded RNA (dsRNA)

47 molecules into small-interfering RNAs (siRNAs) that are 19-25 nucleotides in length; these

48 siRNAs serve as the functional unit of RNAi and govern the location of gene suppression through

49 the degradation of complementary messenger RNA molecules (Fire et al. 1998; Martinez et al.

50 2002; Vermeulen et al. 2005). To date, this process has been investigated in the control of a

51 number of pest groups, including parasites of medical importance, urban pests, pests and

52 pathogens of honey bees, and agricultural pests of economic importance.

53 While the technology promises to be target specific (Whyard et al. 2009; Bachman et al.

54 2013), there is concern that current risk assessment frameworks for genetically modified crops

55 are not adequate to proactively assess the risks to non-target organisms (Romeis et al., 2008;

56 Lundgren and Duan 2013; FIFRA-SAP 2014). The risks associated with RNAi to non-target

57 organisms include immune stimulation (Lu and Liston 2009), saturation of an organism's RNAi

58 machinery that could interfere with normal cellular processes (Grimm 2011; Flenniken and

59 Andino 2013), and unintentional gene silencing. Unintentional gene silencing in non-target

60 organisms is the primary risk posed by pesticidal RNAi; within a non-target species, this

61 unintentional gene silencing can be due to silencing the intended gene in an unintended organism

62 (non-target binding) or silencing a different gene with sufficient sequence similarity to the

63 dsRNA (off-target binding) (Lundgren and Duan 2013; FIFRA-SAP 2014). Because pesticidal 
64 RNAi poses risks to non-target organisms that are different from other pesticides, a risk

65 assessment framework has been proposed to proactively assess these risks using a series of steps

66 (FIFRA-SAP 2014; Roberts et al. 2015). Indeed, the United Nations employs the precautionary

67 principle when conducting risk assessment of genetically modified organisms to ensure that these

68 products do not adversely affect the environment (https://bch.cbd.int/protocol; accessed

69 November 7, 2017).

70 Bioinformatic analyses that compare pesticidal RNAs to non-target genomes can help

71 focus more extensive risk assessment procedures to predict some risks (Heinemann et al., 2013).

72 The hazard to non-target organisms should be predictable if the functional genome of a non-target

73 organism is known, recognizing that numerous circumstances influence gene silencing even

74 when the sequence is identical between a small RNA and the non-target genome (Kerschen et al.

75 2004). Bioinformatic analyses have thus been advocated as an initial screen of potential risks

76 posed by RNAi (FIFRA-SAP 2014; Roberts et al. 2015). In the present study, we used in silico

77 searches to determine whether putative pesticidal dsRNAs share sequence similarities with off-

78 target regions of the honey bee (Apis mellifera L.), a model non-target organism. We were

79 specifically interested in testing the hypotheses that 1) longer dsRNAs increase the potential for

80 off-target binding, 2) non-target silencing of the target gene is dependent on relatedness of the

81 target and non-target species, and 3) certain gene groups in the honey bee are more prone to off-

82 target sequence similarities with pesticidal dsRNAs.

83 Materials and Methods

84 Literature review

85 In broad terms, our approach was to examine the literature for published pesticidal RNAs

86 against an identified suite of pests, and search the targeted gene sequences in the pests for

87 similarities with regions of the honey bee genome. Published studies evaluating the effects of

88 pesticidal dsRNAs were searched using the ISI Web of Knowledge database, using combinations 
89 of the search terms "pesticidal," "insecticidal," "siRNA," "dsRNA," "RNAi," and "RNA

90 interference." See the introduction for a description of these terms. Studies were included if they

91 evaluated the pesticidal effects of a dsRNA or siRNA and provided either the RNA sequence or

92 primer sets that allowed the RNA sequences to be determined from the target species' genome

93 using the NCBI genome database (http:/www.ncbi.nlm.nih.gov/genome/). A total of 24 studies

94 were included, with pesticidal qualities being evaluated for 74 dsRNAs and 21 siRNAs targeting

9557 genes (Supplemental Data 1). These included species of medical importance (Hajdusek et al.

96 2009; Kwon et al. 2013), urban pests (Zhou et al. 2008; Itakura et al. 2009), parasites and

97 pathogens of honey bees (Maori et al. 2009; Campbell et al. 2010; Desai et al. 2012), agricultural

98 pests (Mutti et al. 2006; Baum et al. 2007; Whyard et al. 2009; Tang et al. 2010; Choudhary and

99 Sahi 2011; Wuriyanghan et al. 2011; Gong et al. 2013; Ochoa-Campuzano et al. 2013; Yao et al.

100 2013; Christiaens et al. 2014; Chu et al. 2014; Han et al. 2014; Meng et al. 2014; Miyata et al.

101 2014; Yu et al. 2014), and others (Whyard et al. 2009; Kelkenberg et al. 2015; Petrick et al.

102 2015).

103 In silico sequence similarity identification

104 Published pesticidal dsRNAs ranged from 19 to 2500+ nucleotides in length. These were

105 queried against the annotated honey bee genome accessed through GenBank

106 (http://blast.ncbi.nlm.nih.gov/Blast.cgi) using the BLAST nucleotide algorithm for somewhat

107 similar sequences (blastn). Similar genetic regions were mostly less than $25 \mathrm{nt}$ long, the length

108 expected for active siRNAs randomly generated from a dsRNA molecule. Sequence similarities

109 of 19/21, 20/21, and 21/21 nt were tallied for each RNA against the honey bee genome, and the

110 off-target gene name was recorded. Each off-target gene was only tallied once per dsRNA, even

111 when that dsRNA targeted multiple locations along that gene. Sequence similarity for the target

112 gene (non-target binding) was also recorded. Low quality proteins (as defined by NCBI) and

113 genes of unknown function were excluded from the analysis, as were any regions with high 
114 sequence similarity that did not return any protein or gene information, such that the resultant

115 database represents a conservative estimate of putative binding.

116 Statistical analysis

117 Because data violated parametric assumptions, the number of off-target similarities were

$118 \log (\mathrm{x}+1)$ transformed and dsRNA length were log transformed to uphold assumptions for analysis

119 with linear regression (Systat v.13.1, San Jose, CA, USA). A chi-square test of independence was

120 used to determine whether there was a significant effect of target taxa on the incidence of non-

121 target binding in honey bees, and whether certain functional gene groups were targeted more

122 frequently.

\section{Results and Discussion}

124 dsRNA length-suppression

125 Each of the 74 pesticidal dsRNAs shared at least one region of perfect or high sequence

126 similarity with the honey bee genome (average $28.6 \pm 3.32$ off-target homologies per dsRNA)

127 (Supplemental Data 1). However, none of the published pesticidal siRNAs (21 total, 19-23 nt in

128 length) found sequence similarity within the honey bee genome at our specified level (19/21, $12920 / 21,21 / 21 \mathrm{nt}$ matches), indicating that these much smaller sequences were more specific when

130 focusing on a single non-target organism. This result was mirrored by Li et al. (2015), though 131 siRNAs are not always this benign: Qiu et al. (2005) demonstrated that 5-80\% of tested siRNAs 132 resulted in off-target binding among diverse organisms.

133 Off-target sequence similarity increased significantly as the dsRNA increased in length 134 (linear regression: $\mathrm{F}_{1,100}=623, P<0.001$ ) (Figure 1a), with every increase of $100 \mathrm{nt}$ in the dsRNA resulting in 6 more predicted hits. This strong relationship between dsRNA length and

136 potential off-target binding can be further demonstrated using only the genes described in Miyata 137 et al. (2014), in which the authors evaluated the effects of dsRNA length on RNAi activity in vivo 
138 in western corn rootworms. Although the gene targets in this study were not pesticidal

139 specifically, and thus excluded from our overall analysis, the authors evaluated silencing of the

140 same gene targets (laccase 2 and ebony) using different sized dsRNAs to evaluate efficacy. When

141 we examined this suite of genes from a risk assessment perspective using the same methodology

142 as for the pesticidal RNAs, the longer dsRNAs returned significantly more regions of off-target

143 sequence similarity in the honey bee genome (laccase $2: \mathrm{F}_{1,5}=181, \mathrm{P}<0.001 ;$ ebony: $\mathrm{F}_{1,2}=103$,

$144 \mathrm{P}=0.01$ ) (Figure 1b). While intuitive (Bolognesi et al. 2012), this is the first demonstration of the

145 possibility for increased length-suppression in a non-target organism. Thus, optimizing RNA

146 length to have maximum gene suppression efficacy in the target pest needs to be balanced against

147 the non-target risks posed by longer molecules.

148 Target-species specificity

149 Taxonomic relatedness of the target organism to honey bees had no effect on potential

150 binding of siRNAs on the original gene target (non-target binding) $\left(\chi^{2}=9.4, \mathrm{df}=7, P=0.23\right.$ )

151 (Figure 2). Contrary to assertions of pesticidal specificity (Bachman et al. 2013), this implies that

152 silencing of the target gene in a non-target organism may be more likely to occur from random

153 sequence similarities than based on evolutionary relatedness to the target organism. Although the

154 pool of available literature is limited with regards to targeted applications of RNAi against pest

155 species, with certain species being more frequently researched (e.g. Diabrotica virgifera), our

156 results suggest that non-target hazard assessments should focus on species of ecological relevance

157 rather than strictly on phylogenetic relatedness to the target species.

158 Unfortunately, when conducting bioinformatics analyses for the purposes of a risk

159 assessment, the availability of sequenced genomes from representative species becomes a

160 limiting factor. Further, the potential non-target community will differ depending on the specific

161 pest being targeted, making it difficult to have a standard suite of species to evaluate for non-

162 target effects. Bioinventories are crucial for identifying appropriate non-target species for each 
163 target pest. Supporting initiatives such as i5K (i5K Consortium 2013), which strives to sequence

164 the genomes of 5000 representative invertebrates, and making these genomes freely available,

165 will bolster the applicability of future in silico analyses aimed at identifying potential risks of

166 gene-oriented pest control.

167 Targeted gene groups

168 The homeobox genes and other genes involved in embryonic and developmental

169 pathways in honey bees frequently shared sequence similarity with the pesticidal dsRNAs,

170 particularly when vATPase subunits were the pesticidal targets $\left(\chi^{2}=10\right.$, df $\left.=4, P=0.03\right) .67 \%$ of

171 all tested dsRNAs had the high potential to bind to honey bee developmental genes that were not

172 the target, and 33\% of these shared high similarity with homeobox genes specifically

173 (Supplemental Data 1). Although we have an incomplete picture of which genes are expressed in

174 most genomes at any given time, many of these genes, while important during embryogenesis

175 and development, support additional critical functions such as cell proliferation and apoptosis,

176 and are highly conserved across metazoans. In this instance, in silico analysis identified some of

177 the potential gene targets that could present a hazard requiring unique assessments across life

178 stages to properly identify a phenotypic effect. If validated in future in vivo assessments, this

179 screening method may prove useful in identifying appropriate experimental endpoints in non180 target risk assessments.

\section{Conclusions}

Our bioinformatics-based in silico analysis provides a conservative assessment of

183 potential off-target binding of pesticidal dsRNAs in the honey bee genome; the actual binding

184 affinity of RISC is more nuanced than $100 \%$ or similar sequence similarity for subsequent mRNA

185 degradation. While some have documented suppression of off-target gene expression with 20/21

186 nt similarity (Jarosch and Moritz 2012), others have found silencing with even less sequence 
187 similarity in certain study systems, particularly in the 2-8 nt seed region of the siRNA. For

188 example, in experiments with cultured human cells, Saxena et al. (2003) found gene silencing

189 with as many as 3-4 bp mismatches in addition to G.U wobbles (guanine and uracil have a slight

190 affinity for each other), while Jackson et al. (2003) found mRNA degradation with only 11/21

191 contiguous nt. The locations of the mismatches along the siRNA are also important; perfect

192 sequence similarity of the seed region is particularly crucial for mRNA recognition (Jackson et al.

193 2006; Chu et al. 2014). Additionally, the off-target effects may not be easily predicted, as

194 downstream effects may result in gene suppression unrelated to the sequence of the original

195 pesticidal molecule (Hanning et al., 2013). Another area of research that merits attention from a

196 risk assessment perspective is that of RNA replication of the primary pesticidal molecule within a

197 non-target organism. It has been repeatedly shown that environmental RNAi is replicated within a

198 cell, and that the secondary RNAs produced do not always perfectly match the original sequence

199 of the insecticidal RNA (Pak and Fire, 2007; Sijen et al., 2007). Focusing in silico analyses on

200 only the primary insecticidal molecule may overlook these potential non-target effects.

201

However, in silico identification of sequence similarity between a pesticidal dsRNA and

non-target organism's genome does not imply that RNAi will occur in the non-target organism. A

203 fundamental difference between RNAi and chemical pesticides resides in their spectrum and

204 mode of activity. Arguably, biochemical pesticides work on a limited number of physiological

205 targets within an organism, and the list of potential non-target species is restricted to those

206 sharing these targets with the pest. The absence of a relationship of taxonomic relatedness of

207 target and off-target species and the likelihood of gene similarity between them indicates that the

208 list of species potentially at risk from RNAi initially includes all of those species that use mRNA

209 for gene expression and have the cellular machinery to process small RNAs. This spectrum of

210 activity, and broad set of potential unintended phenotypic effects of the pesticidal RNAi may

211 make predicting the risk of this technology more challenging than other pesticides. Unintended 
212 gene silencing will depend on a number of factors. The organism would need to possess

213 behavioral characteristics that would put it into contact with contaminated materials, e.g. leaf

214 tissue versus pollen versus nectar feeding at a contaminated location. Other factors include the

215 length of the dsRNA and whether the organism is exposed to siRNA or dsRNA, the identity of

216 the target or off-target mRNA, the size of a non-target organism's genome (more off-target

217 binding would be expected when there are more potential gene targets), the necessary binding

218 affinity of a particular siRNA, exposure concentration of the dsRNA, and the physiological state

219 of the insect (Qiu et al. 2005; Baum et al. 2007; Huvenne and Smagghe 2010; Gu et al. 2014).

220 Ecological risk assessment is a complex and multi-stepped process, and no single piece of

221 work is sufficient to fully quantify the risk of a toxicological event. We have demonstrated that an

222 in silico analysis may be used as a first step in establishing whether off-target binding could pose

223 a significant threat for a particular pesticidal dsRNA in a non-target organism such as the honey

224 bee. Future experiments to evaluate the usefulness of this tool are planned that would quantify

225 up/down gene regulation of honey bees exposed to pesticidal dsRNA. Taken together, these data

226 may provide a basis for designing biologically appropriate experiments to optimize hazard

227 assessments for applications of this novel pesticidal technology in field settings where honey bees

228 and other non-target organisms may be exposed.

\section{Acknowledgements}

230 We thank Casey Snyder and Nathan Koens for assisting in compiling the RNAi database.

231 Mention of trade names or commercial products is solely for the purpose of providing specific

232 information and does not imply recommendation or endorsement by the U.S. Department of 233 Agriculture. 
234

235 236

237

238

239

240

241

242

243

244

245

246

247

248

249

250

251

252

253

254

255

256

257

258

\section{References}

Bachman, P. M., Bolognesi, R., Moar, W. J., Mueller, G. M., Paradise, M. S., Ramaseshadri, P., Tan, J., Uffman, J. P., Warren, J., Wiggins, B. E., Levine, S. L. (2013) Characterization of the spectrum of insecticidal activity of a double-stranded RNA with targeted activity against Western Corn Rootworm (Diabrotica virgifera virgifera LeConte). Transgenic Res 22(6): $1207-1222$.

Baum, J. A., Bogaert, T., Clinton, W., Heck, G. R., Feldmann, P., Ilagan, O., Johnson, S. D., Plaetinck, G., Munyikwa, T., Pleau, M., Vaughn, T., Roberts, J. (2007) Control of coleopteran insect pests through RNA interference. Nat Biotechnol 25(11): 1322-1326.

Bolognesi, R., Ramaseshadri, P., Anderson, J., Bachman, P., Clinton, W., Flannagan, R., Ilagan, O., Lawrence, C., Levine, S., Moar, W., Mueller, G., Tan, J., Uffman, J. Wiggins, E., Heck, G., Segers, G. (2012) Characterizing the mechanism of action of double-stranded RNA activity against Western Corn Rootworm (Diabrotica virgifera virgifera LeConte). PLoS One 7(10): e47534.

Campbell, E. M., Budge, G. E., Bowman, A. S. (2010) Gene-knockdown in the honey bee mite Varroa destructor by a non-invasive approach: studies on a glutathione S-transferase. Parasite Vector 3: 73 .

Choudhary, M., Sahi, S. (2011) In silico designing of insecticidal small interfering RNA (siRNA) for Helicoverpa armigera control. Indian J Exp Biol 49(6): 469-474.

Christiaens, O., Swevers, L., Smagghe, G. (2014) dsRNA degradation in the pea aphid (Acyrthosiphon pisum) associated with lack of response in RNAi feeding and injection assay. Peptides 53: 307-314.

Chu, C.-C., Sun, W., Spencer, J. L., Pittendrigh, B. R., Seufferheld, M. J. (2014) Differential effects of RNAi treatments on field populations of the western corn rootworm. Pestic Biochem Phys 110: 1-6. 
259 Desai, S. D., Eu, Y. J., Whyard, S., Currie, R. W. (2012) Reduction in deformed wing virus

260 infection in larval and adult honey bees (Apis mellifera L.) by double-stranded RNA

261 ingestion. Insect Mol Biol 21(4): 446-455.

262 FIFRA-SAP. (2014) RNAi technology: Program formulation for human health and ecological

263 risk assessment. FIFRA Scientific Advisory Panel Minutes No. 2014-2.

264 Fire, A., Xu, S. Q., Montgomery, M. K., Kostas, S. A., Driver, S. E., Mello, C. C. (1998) Potent

265 and specific genetic interference by double-stranded RNA in Caenorhabditis elegans. Nature 266 391(6669): 806-811.

267 Flenniken, M. L., Andino, R. (2013) Non-specific dsRNA-mediated antiviral response in the 268 honey bee. PLoS One 8(10): e77263.

269 Gong, L., Chen, Y., Hu, Z., Hu, M. (2013) Testing insecticidal activity of novel chemically 270 synthesized siRNA against Plutella xylostella under laboratory and field conditions. PLoS 271 One 8(5): e62990.

272 Grimm, D. (2011) The dose can make the poison: lessons learned from adverse in vivo toxicities 273 caused by RNAi overexpression. Science 2: 8-8.

274 Gu, L., Knipple, D. C. (2013) Recent advances in RNA interference research in insects:

275 Implications for future insect pest management strategies. Crop Prot 45: 36-40.

276 Gu, S., Zhang, Y., Jin, L., Huang, Y., Zhang, F., Bassik, M. C., Kampmann, M., Kay, M. A.

277 (2014) Weak base pairing in both seed and 3' regions reduces RNAi off-targets and enhances 278 si/shRNA designs. Nucleic Acids Res 42(19): 12169-12176.

279 Hajdusek, O., Sojka, D., Kopacek, P., Buresova, V., Franta, Z., Sauman, I., Winzerling, J., 280 Grubhoffer, L. (2009) Knockdown of proteins involved in iron metabolism limits tick reproduction and development. Proc Natl Acad Sci USA 106(4): 1033-1038. 
282 Hajeri, S., Killiny, N., El-Mohtar, C., Dawson, W. O., Gowda, S. (2014) Citrus tristeza virus283 based RNAi in citrus plants induces gene silencing in Diaphorina citri, a phloem-sap sucking insect vector of citrus greening disease (Huanglongbing). J Biotechnol 176: 42-49.

285

Han, P., Fan, J., Liu, Y., Cuthbertson, A. G. S., Yan, S., Qiu, B.-L., Ren, S. (2014) RNAimediated knockdown of serine protease inhibitor genes increases the mortality of Plutella xylostella challenged by destruxin A. PLoS One 9(5).

Hanning, J.E., Saini, H. K. , Murray, M. J., van Dongen, S., Davis, M. P. A., Barker, E. M, Ward, D. M., Scarpini, C. G., Enright, A. J., Pett, M. R., Coleman, N. (2013) Lack of correlation between predicted and actual off-target effects of short-interfering RNAs targeting the human papillomavirus type 16 E7 oncogene. Br J Cancer 108: 450-460.

Heinemann, J. A., Agapito-Tenfen, S. Z., Carman, J. A. (2013) A comparative evaluation of the regulation of GM crops or products containing dsRNA and suggested improvements to risk assessments. Environ Int 55: 43-55.

Huvenne, H., Smagghe, G. (2010) Mechanisms of dsRNA uptake in insects and potential of RNAi for pest control: A review. J Insect Physiol 56(3): 227-235.

i5K Consortium. (2013) The i5K Initiative: advancing arthropod genomics for knowledge, human health, agriculture, and the environment. J Hered 104(5): 595-600.

Itakura, S., Murayama, S., Kamata, Y., Tanaka, H., Enoki, A. (2009) RNA interference in symbiotic protists of the termite Coptotermes formosanus (Isoptera: Rhinotermitidae) through ingestion of siRNA by the host termite. Sociobiology 54(1): 77-87.

Jackson, A. L., Bartz, S. R., Schelter, J., Kobayashi, S. V., Burchard, J., Mao, M. K., Li, B., Cavet, G., Linsley, P. S. (2003) Expression profiling reveals off-target gene regulation by RNAi. Nat Biotechnol 21(6): 635-637. 
305 Jackson, A. L., Burchard, J., Leake, D., Reynolds, A., Schelter, J., Chau, B. N., Cleary, M., Lim, 306 L., Linsley, P. S. (2006) Position-specific chemical modification of siRNAs reduces "off307 target" transcript silencing. RNA 12(7): 1197-1205.

308 Jarosch, A., Moritz, R. F. A. (2012) RNA interference in honeybees: off-target effects caused by 309 dsRNA. Apidologie 43(2): 128-138.

310 Kelkenberg, M., Odman-Naresh, J., Muthukrishnan, S., Merzendorfer, H. (2015) Chitin is a 311 necessary component to maintain the barrier function of the peritrophic matrix in the insect 312 midgut. Insect Biochem Mol Biol 56: 21-28.

313 Kerschen, A., Napoli, C. A., Jorgensen, R. A., Müller, A. E. (2004) Effectiveness of RNA 314 interference in transgenic plants. FEBS Letters 566: 223-228.

315 Kwon, D. H., Park, J. H., Lee, S. H. (2013) Screening of lethal genes for feeding RNAi by leaf 316 disc-mediated systematic delivery of dsRNA in Tetranychus urticae. Pestic Biochem Phys $317 \quad 105(1): 69-75$.

Li, H., Khajuria, C., Rangasamy, M., Gandra, P., Fitter, M., Geng, C., Woosely, A., Hasler, J., 319 Schulenberg, G., Worden, S., McEwan, R., Evans, C., Siegfied, B., Narva, K. E. (2015) Long dsRNA but not siRNA initiates RNAi in western corn rootworm larvae and adults. J Appl Entomol 139(6): 432-445.

Lu, L.-F., Liston, A. (2009) MicroRNA in the immune system, microRNA as an immune system. Immunology 127(3): 291-298.

Lundgren, J. G., Duan, J. J. (2013) RNAi-based insecticidal crops: Potential effects on nontarget species. BioScience 63(8): 657-665.

Maori, E., Paldi, N., Shafir, S., Kalev, H., Tsur, E., Glick, E., Sela, I. (2009) IAPV, a bee-affecting virus associated with Colony Collapse Disorder can be silenced by dsRNA ingestion. Insect Mol Biol 18(1): 55-60. 
329

330 331

Marr, E. J., Sargison, N. D., Nisbet, A. J., Burgess, S. T. G. (2014) RNA interference for the identification of ectoparasite vaccine candidates. Parasite Immunol 36(11): 614-624.

Martinez, J., Patkaniowska, A., Urlaub, H., Luhrmann, R., Tuschl, T. (2002) Single-stranded antisense siRNAs guide target RNA cleavage in RNAi. Cell 110(5): 563-574.

Meng, X., Li, L. M., Gao, G., Jin, F. L., Ren, S. X. (2014) The gene expression of the protein SLAWD, mediating the toxic effect of destruxin A on Spodoptera litura larvae, in prokaryotic cells: purification and characterization. Mol Biol 48(6): 908-914.

Miyata, K., Ramaseshadri, P., Zhang, Y., Segers, G., Bolognesi, R., Tomoyasu, Y. (2014) Establishing an in vivo assay system to identify components involved in environmental RNA interference in the western corn rootworm. PLoS One 9(7): e101661.

Mutti, N. S., Park, Y., Reese, J. C., Reeck, G. R. (2006) RNAi knockdown of a salivary transcript leading to lethality in the pea aphid, Acyrthosiphon pisum. J Insect Sci 6: 1-7.

Ochoa-Campuzano, C., Martinez-Ramirez, A. C., Contreras, E., Rausell, C., Real, M. D. (2013) Prohibitin, an essential protein for Colorado potato beetle larval viability, is relevant to Bacillus thuringiensis Cry3Aa toxicity. Pestic Biochem Phys 107(3): 299-308.

Pak, J., Fire, A. (2007) Distinct populations of primary and secondary effectors during RNAi in C. elegans. Science 315: 241-244.

Petrick, J. S., Moore, W. M., Heydens, W. F., Koch, M. S., Sherman, J. H., Lemke, S. L. (2015) A 28-day oral toxicity evaluation of small interfering RNAs and a long double-stranded RNA targeting vacuolar ATPase in mice. Regul Toxicol Pharm 71(1): 8-23.

Price, D. R. G., Gatehouse, J. A. (2008) RNAi-mediated crop protection against insects. Trends Biotechnol 26(7): 393-400.

Qiu, S., Adema, C. M., Lane, T. (2005) A computational study of off-target effects of RNA interference. Nucleic Acids Res 33(6): 1834-1847. 
353 Roberts, A. F., Devos, Y., Lemgo, G. N. Y., Zhou, X. (2015) Biosafety research for non-target

354 organism risk assessment of RNAi-based GE plants. Front Plant Sci 6: Article 958.

355 Romeis, J., Bartsch, D., Bigler, F., Candolfi, M. P., Gielkens, M. M. C., Hartley, S. E., Hellmich,

Sijen, T., Steiner, F. A., Thijssen, K. L., Plasterk, R. H. (2007) Secondary siRNAs result from unprimed RNA synthesis and form a distinct class. Science 315: 244-247.

Tang, B., Wang, S., Zhang, F. (2010) Two storage hexamerins from the beet armyworm Spodoptera exigua: Cloning, characterization and the effect of gene silencing on survival. BMC Mol Biol 11: 65.

Vermeulen, A., Behlen, L., Reynolds, A., Wolfson, A., Marshall, W. S., Karpilow, J., Khvorova, A. (2005) The contributions of dsRNA structure to Dicer specificity and efficiency. RNA 11(5): 674-682.

Whyard, S., Singh, A. D., Wong, S. (2009) Ingested double-stranded RNAs can act as speciesspecific insecticides. Insect Biochem Mol Biol 39(11): 824-832.

Wuriyanghan, H., Rosa, C., Falk, B. W. (2011) Oral delivery of double-stranded RNAs and siRNAs induces RNAi effects in the potato/tomato psyllid, Bactericerca cockerelli. PLoS One 6(11): e27736.

Yao, J., Rotenberg, D., Afsharifar, A., Barandoc-Alviar, K., Whitfield, A. E. (2013) Development of RNAi methods for Peregrinus maidis, the corn planthopper. PLoS One 8(8): e70243. 
378 Yu, R., Xu, X., Liang, Y., Tian, H., Pan, Z., Jin, S., Wang, N., Zhang, W. (2014) The insect 379 ecdysone receptor is a good potential target for RNAi-based pest control. Int J Biol Sci $380 \quad 10(10): 1171-1180$.

381 Zhou, X., Wheeler, M. M., Oi, F. M., Scharf, M. E. (2008) RNA interference in the termite 382 Reticulitermes flavipes through ingestion of double-stranded RNA. Insect Biochem Mol Biol $383 \quad 38(8): 805-815$.

Figure 1. The relationship between pesticidal dsRNA length and potential off-target binding in honey bees for pesticidal dsRNAs (a) and the non-pesticidal laccase 2 and ebony genes (data 387 from Miyata et al. (2014)) (b). versus the original target taxa (x-axis), in relation to the total number of examined pesticidal dsRNAs. Taxa are ordered by increasing relative divergence time from honey bees. 
Figure 1

Pesticidal dsRNA length and potential off-target binding in honey bees

The relationship between pesticidal dsRNA length and potential off-target binding in honey bees for pesticidal dsRNAs (a) and the non-pesticidal laccase 2 and ebony genes (data from Miyata et al. (2014)) (b).

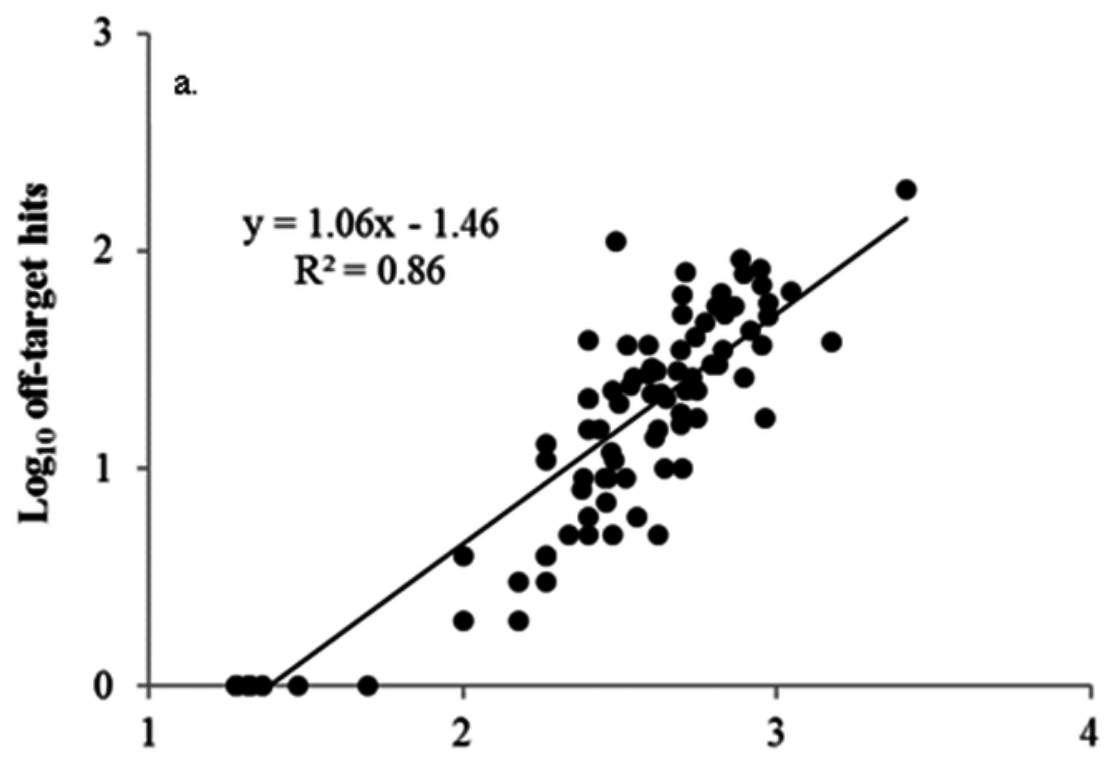

$\log _{10}$ length of pesticidal dsRNA

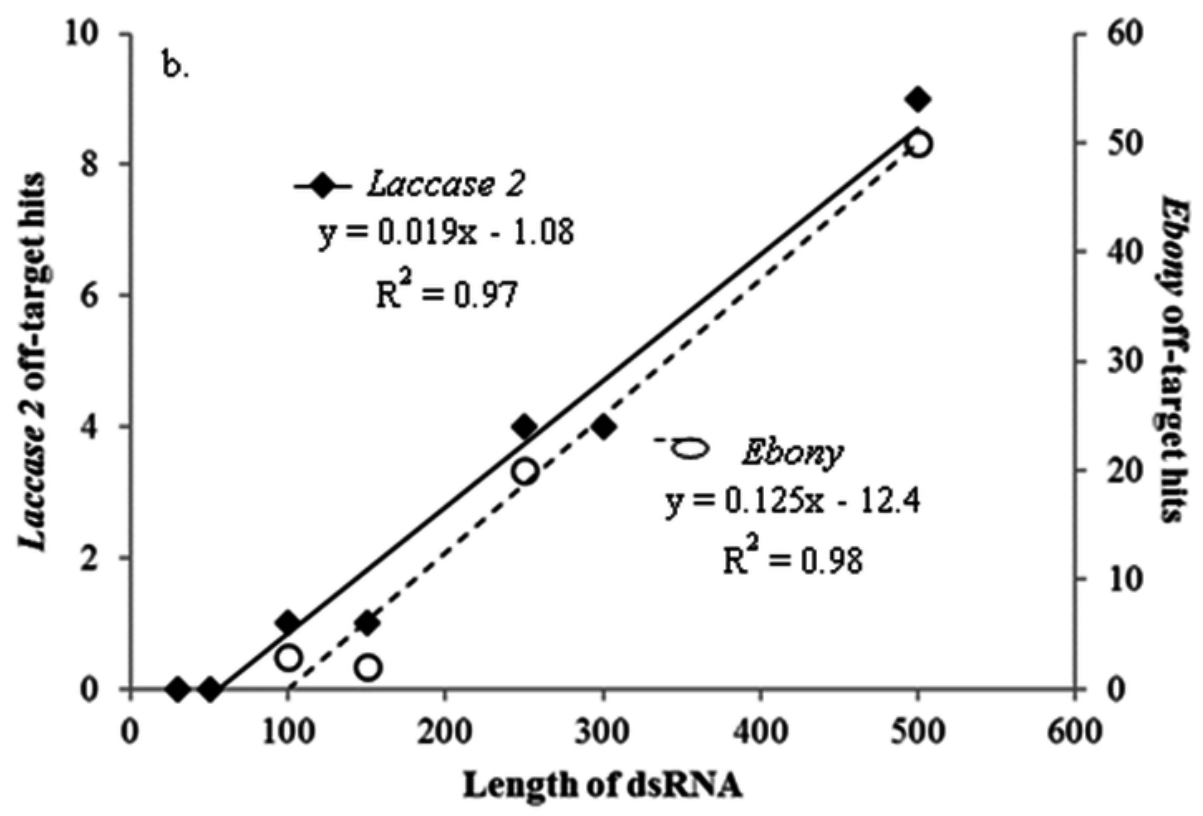


Figure 2

Pesticidal dsRNA target organisms and the likelihood of off-target binding in the honey bee genome.

Potential non-target binding of pesticidal dsRNAs in honey bees (y-axis, shaded area) versus the original target taxa ( $\mathrm{x}$-axis), in relation to the total number of examined pesticidal dsRNAs. Taxa are ordered by increasing relative divergence time from honey bees. 


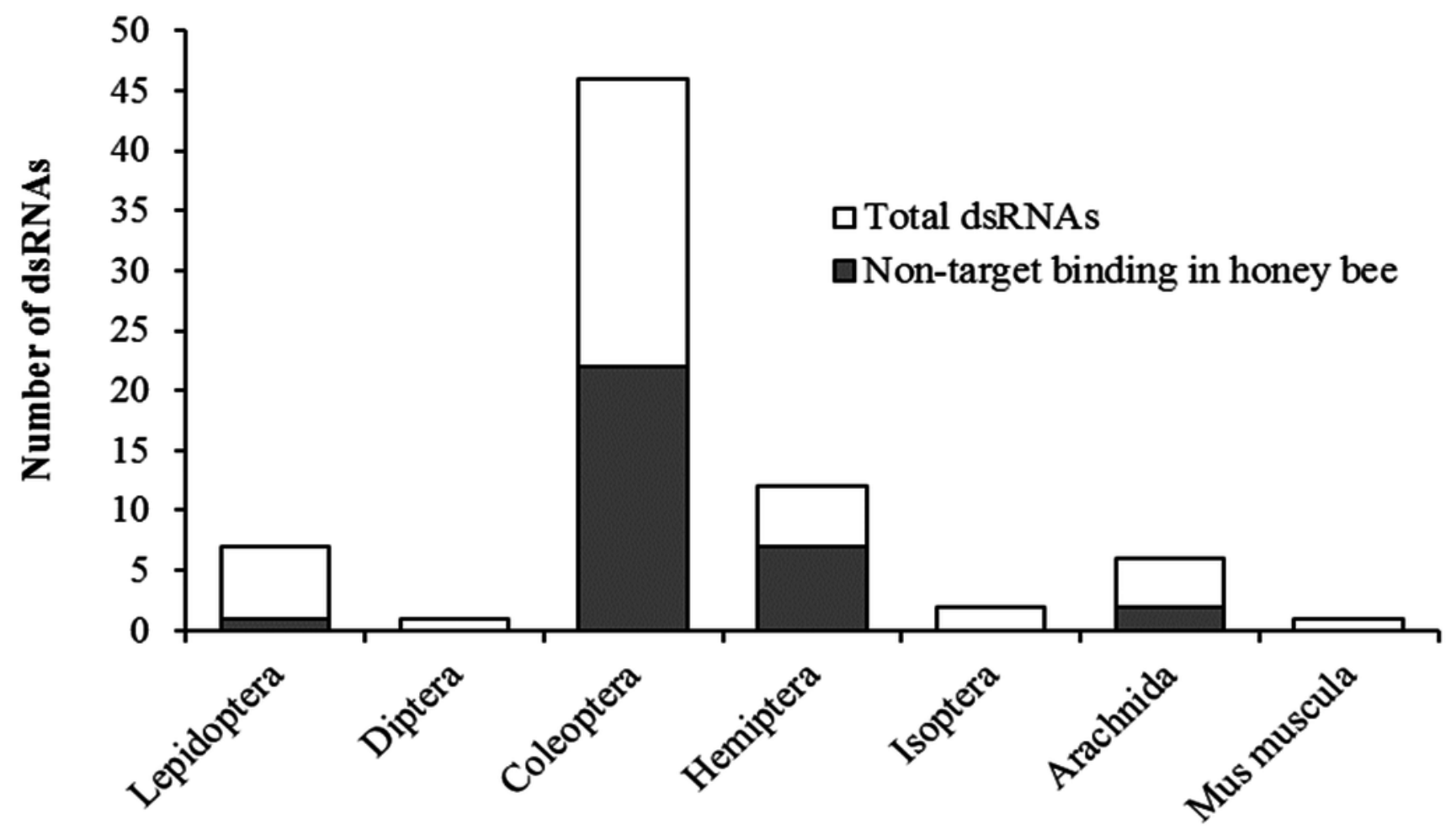

